\begin{tabular}{|l|c|c|c|c|}
\hline $\begin{array}{l}\text { Cuadernos de Investigación Geográfica } \\
\text { Geographical Research Letters }\end{array}$ & 2019 & N $^{\circ} 45(2)$ & pp. 709-728 & eISSN 1697-9540 \\
\hline
\end{tabular}

\title{
IDENTIFICACIÓN DE CONFLICTOS DE USO DE SUELO PARA LA PLANIFICACIÓN DEL CRECIMIENTO URBANO: CIUDAD CUAUHTÉMOC, CHIHUAHUA (MÉXICO)
}

\author{
J.D. MALDONADO-MARÍN, L.C. ALATORRE-CEJUDO, \\ E. SÁNCHEZ-FLORES
}

Instituto de Arquitectura Diseño y Arte, Universidad Autónoma de Ciudad Juárez, Av. del Charro 450 N, 32310 Ciudad Juárez, Chihuahua, México.

\begin{abstract}
RESUMEN. Esta investigación incorpora nuevas formas de análisis para la planificación y desarrollo urbano en Ciudad Cuauhtémoc, Chihuahua (México), aportando elementos de referencia mediante la identificación de zonas con potencialidad y limitaciones para el uso de suelo urbano, así como para las actividades agrarias y de conservación. El objetivo general fue identificar los principales conflictos entre los usos y coberturas de suelo para determinar las áreas de mayor aptitud territorial para el crecimiento de la ciudad. Para este propósito se utilizó el modelo Land Use Conflict Identification Strategy (LUCIS) con el fin de entender el significado espacial del estatus de las políticas de uso de suelo, incluyendo los patrones urbanos probables asociados con las tendencias agrarias $y$ de conservación. En el caso de estudio se estima un total de 149.139 habitantes para el año 2030, lo que representa la necesidad de 392,42 hectáreas adicionales para albergar el crecimiento poblacional. Para ello de las 16.272,21 hectáreas que tiene el límite de población, el 38\% se asignaron a la categoría de agraria, el 11,95\% a suelos de conservación y el 49,67\% a suelo urbano (incluyendo el área urbana existente). Existe una porción importante del área que se encuentra en conflicto entre los diferentes usos de suelo. Se concluye que la integración de un modelo de resolución de conflictos de cobertura y uso de suelo representa una solución práctica que contribuye a la mejora de los procesos de planificación del desarrollo urbano.
\end{abstract}

Identification of land use conflicts for urban growth planning: Ciudad Cuauhtémoc, Chihuahua (Mexico)

ABSTRACT. This research incorporates new forms of analysis for urban planning and development in Ciudad Cuauhtémoc, Chihuahua (Mexico), providing elements of reference by identifying areas with potentiality and limitations for urban land use, as well as for agricultural and conservation activities. The general objective was to identify the main conflicts between land uses and coverages to determine the areas of greatest territorial suitability for the city's growth. For this purpose, the Land Use Conflict Identification Strategy (LUCIS) model was used 
to understand the spatial significance of the status of land use policies, including likely urban patterns associated with agricultural and conservation trends. In the case study, a total of 149,139 inhabitants are estimated for the year 2030, which represents the need for an additional 392.42 hectares to accommodate the population growth. For that of the 16,272.21 hectares that has the population limit, $38 \%$ were allocated to the category of agriculture, $11.95 \%$ to conservation soils and $49.67 \%$ to urban land (including the existing urban area). There is a significant portion of the area that is in conflict between the different land uses. It concludes, that the integration of a conflict resolution model for land use and land cover represents a practical solution that contributes to the improvement of processes of urban development planning.

Palabras clave: aptitud territorial, planificación urbana, análisis espacial, conflicto entre usos de suelo, SIG.

Key words: land suitability, urban planning, spatial analysis, land use conflict, GIS.

*Correspondencia: Juan Diego Maldonado Marín, Instituto de Arquitectura Diseño y Arte, Universidad Autónoma de Ciudad Juárez, Av. del Charro 450 N, C.P. 32310 , Ciudad Juárez, Chihuahua, México. E-mail: juan.dimm.130993@gmail.com

\section{Introducción}

La urbanización de la población a nivel mundial ha provocado el crecimiento acelerado de las ciudades en el último siglo (Rionda, 2008; da Cunha y Rodríguez, 2009), con la consecuente presión sobre los recursos naturales, evidenciando una fuerte competencia entre los usos de agua, suelos agrícolas y urbanos (Díaz et al., 2014; Alatorre et al., 2014). La explosión demográfica y el prevaleciente desequilibrio en la distribución de ingresos a nivel nacional han propiciado las condiciones para una expansión urbana anárquica en muchas ciudades de México, debido a que las tierras agrícolas periurbanas han dejado de producir y se han vuelto objeto de especulación (Bazant, 2010).

Al final del siglo XX se presentó una transformación en la sociedad occidental. Debido a la modernización económica acelerada se ha generado una marcada pobreza urbana, con un régimen de desigualdad y marginalidad (Wacquant, 2001). México sigue urbanizándose significativamente, además de presentar desigualdades regionales de crecimiento urbano ya que diversos factores influyeron en la distribución territorial de las actividades económicas (Garza, 2010).

La planificación territorial como respuesta institucional a los procesos de expansión urbana se establece así como un proceso para determinar acciones asociadas para el futuro a través de una secuencia de alternativas (Davidoff y Reiner, 1962), primero seleccionando fines y criterios, luego identificando un conjunto de alternativas, para finalmente orientar la acción a una alternativa deseada. Desde un enfoque racional, este proceso 
implica adoptar y producir planes y políticas públicas en las que el planificador genera conocimiento para entender el problema y proponer soluciones al mismo (Peña, 2016).

En el caso de Ciudad Cuauhtémoc, en el estado de Chihuahua (México), ha presentado un crecimiento poblacional acelerado en las últimas décadas, convirtiéndose en una de las ciudades más pobladas del estado, con un crecimiento muy por encima de la media municipal en el Estado (INEGI, 2015). Por esta razón se ha establecido la prioridad de evaluar las mejores alternativas para un crecimiento planificado que considere el potencial del territorio. El uso de modelos espaciales, basados en técnicas de análisis multicriterio constituye una gran alternativa metodológica para tal objetivo (Molero et al., 2007). Estas herramientas pueden ayudar a identificar los mejores lugares para la planificación de nuevas áreas de crecimiento urbano (Daga, 2009). Además, identificar los factores geográficos que intervienen en el proceso es de gran importancia, ya que en la actualidad la protección del medio ambiente y la conservación de los recursos se han convertido en una necesidad fundamental que forma parte central de los procesos de planificación (Silberstein y Maser, 2013).

Aunado a todo esto, el Gobierno Municipal de Cuauhtémoc no cuenta con un Instituto de Planeación Urbana, y además el Plan de Desarrollo Urbano (PDU) no se actualiza de acuerdo con las necesidades y el crecimiento de la ciudad. Estos planes carecen de un marco metodológico y se han orientado principalmente a realizar una descripción de los recursos con los que se cuenta. El PDU presenta una propuesta de zonificación en la que el área urbana para el año 2008 era de 3091,79 ha, mientras que el área de reserva para crecimiento al año 2027 se establecía en 3262,70 ha, con una reserva ecológica de 9921,56 ha, considerando una superficie total del centro de población en sus límites de expansión de 16.276,05 ha (SDUE; Gob. Municipal de Cuauhtémoc, 2009). Sin embargo, el problema radica en que no se especifican los criterios utilizados en la selección de las áreas de reserva para el crecimiento urbano, lo que abre la posibilidad de que las nuevas zonas de expansión se hayan seleccionado sin tomar en cuenta la aptitud física, económica y social del territorio para alojar el crecimiento.

La competencia por el suelo para encontrar espacios con mayores ventajas económicas, elementos naturales favorables, además de accesibilidad a servicios educativos, de salud, culturales y administrativos, ha acelerado el fenómeno del crecimiento de las ciudades (Cifuentes, 2009). Considerando esta competencia por el suelo, en este estudio se plantea como objetivo general identificar los principales conflictos entre los usos y coberturas de suelo que determinan las áreas de mayor aptitud territorial para el crecimiento urbano de Ciudad Cuauhtémoc, Chihuahua. Como objetivos específicos se establece: A) Definir y asignar la idoneidad del uso de suelo para la categoría agrario, urbano y conservación; B) Proponer una asignación del uso y cobertura de suelo de cara al futuro.

\section{2. Área de estudio}

Comprende el límite del Centro de Población de Ciudad Cuauhtémoc, Chihuahua (México), establecido de acuerdo con lo propuesto en el Plan de Desarrollo Urbano en el año 2009. Presenta una superficie de 16.276,05 ha, una población de 117.760 habitantes en 
el año 2010 y un área urbana de 32 km²(Fig. 1). El crecimiento en Ciudad Cuauhtémoc se ha influenciado por las actividades económicas que se desarrollan en la región, principalmente en agricultura, ganadería y fruticultura, seguido de la industria y el comercio.

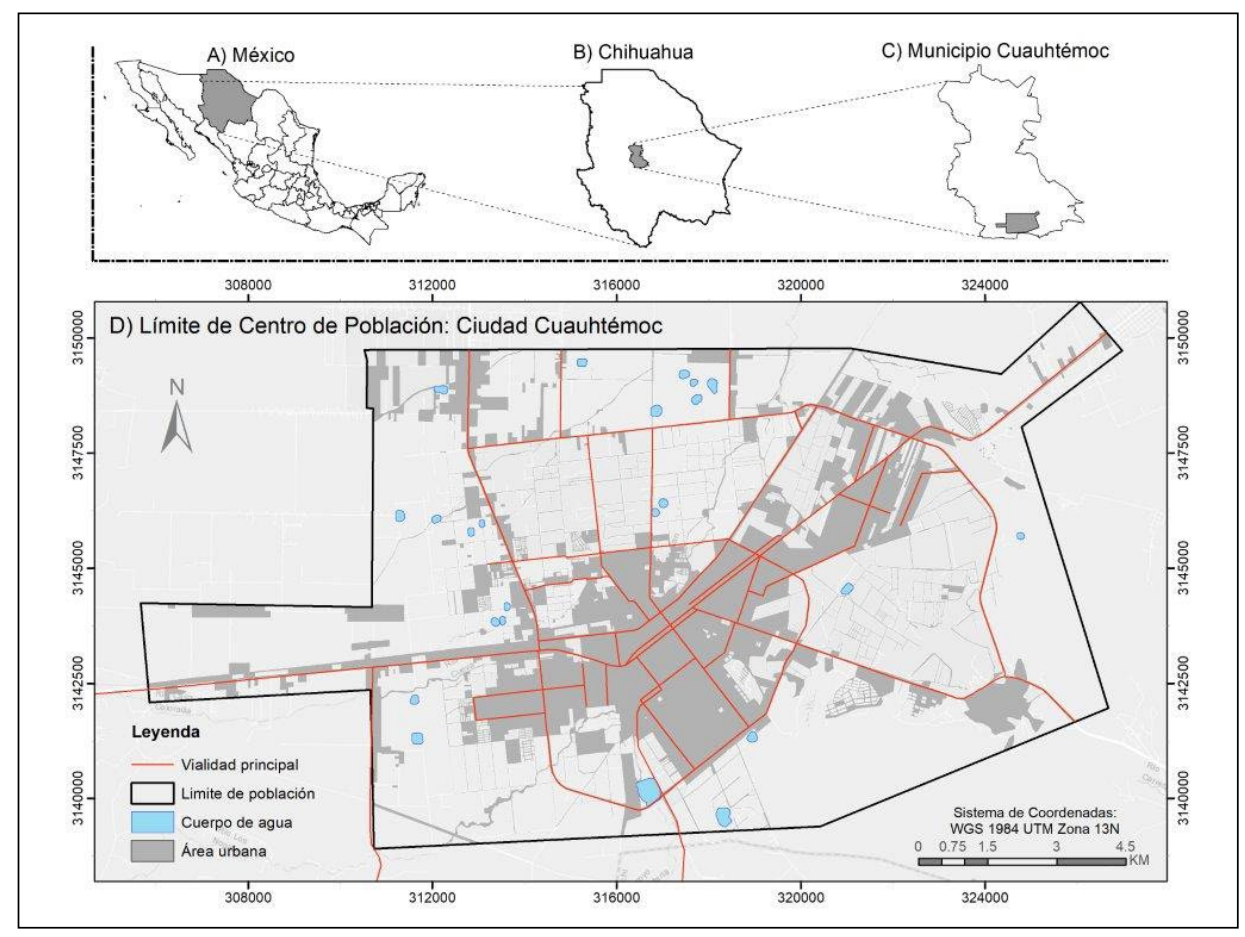

Figura 1. Límite de Centro de Población de Ciudad Cuauhtémoc, Chihuahua, México.

La expansión urbana de Ciudad Cuauhtémoc, a partir del año 1990, se ha generado principalmente sobre las áreas con alguna vía de comunicación importante. El conflicto generado en el área peri-urbana de la ciudad está ligado a las tensiones que surgen entre usuarios urbanos y agrícolas por la expansión del área urbana. En la zona sureste se encuentra una región de montañas que impide el crecimiento en esas áreas, mientras que al norte y hacia el este se localizan las huertas manzaneras. La opción para la expansión se sitúa en la zona noreste y oeste de la ciudad, donde existen las propiedades privadas ya sea por parte de ejidos o comunidades menonitas. Este tipo de conflictos son los que impiden una expansión urbana equilibrada y no permiten generar áreas de reserva territorial para dicho crecimiento.

\section{Métodos}

El método empleado se dividió en una fase descriptiva, una de diagnóstico y otra de gestión de la información; identificando a partir de éstas los efectos locales sobre el medio físico o la sociedad. Partiendo de la integración de las bases de datos 
espaciales fue posible seleccionar las variables que permitieron crear rangos e índices espacialmente distribuidos sobre el área de estudio para, posteriormente, a través de árboles de decisión, generar diversos mapas para la obtención de un modelo general con las mejores zonas para el desarrollo urbano, agrario y de conservación en Ciudad Cuauhtémoc.

\subsection{Modelo LUCIS: Land-Use Conflict Identification Strategy}

LUCIS es un modelo que utiliza el geoprocesamiento basado en sistemas de información geográfica como base para proyectar las futuras alternativas de uso del suelo (Carr y Zwick, 2007). Este método se considera adecuado para analizar la idoneidad y la preferencia para las actividades agrarias, urbanas y de conservación, determinando el potencial de futuros conflictos y construyendo escenarios de uso de suelo. LUCIS produce una representación espacial de patrones probables de uso de suelo dentro de las categorías:

- Suelos de conservación existentes

- Suelos urbanos existentes

- Suelos agrarios existentes

- Áreas preferentes para el uso futuro de suelos agrarios

- Áreas preferentes para el uso futuro de suelos de conservación

- Áreas preferentes para el uso futuro de suelos urbanos

- Áreas de conflicto futuro entre usos de suelo agrario y de conservación

- Áreas de conflicto futuro entre usos de suelo agrario y urbano

- Áreas de conflicto futuro entre usos de suelo urbano y de conservación

- Áreas de conflicto futuro entre usos de suelo agrario, de conservación y urbano

La estrategia se basó en identificar la idoneidad, preferencia y conflicto generados entre los usos del suelo. La idoneidad se consideró como una medida del grado relativo en que una unidad de suelo es apta para un propósito específico. La preferencia es el grado en que una categoría de uso de suelo (agrario, urbano, conservación) es predominante para una unidad de suelo. Finalmente, el conflicto del uso de suelo se identificó por la comparación de la preferencia derivada de cada categoría con las unidades de cada uso de suelo (Carr y Zwick, 2007). Para el desarrollo del modelo fue necesario determinar: 1) Metas y objetivos; 2) Inventario de datos; 3 ) Idoneidad; 4) Preferencia; y 5) Conflicto.

\subsubsection{Metas y objetivos}

El primer paso fue definir las metas, objetivos y subobjetivos para el caso de estudio de Ciudad Cuauhtémoc, en las categorías de uso de suelo para determinar usos preferentes: conservación, urbano y agrario. Esto tuvo como propósito comparar los resultados 
preferentes para definir la localización más adecuada a partir de los conflictos entre los diferentes usos. Las metas fueron establecidas a partir de lo señalado en el modelo LUCIS, adecuado a la región de estudio, tal como se describe en la Tabla 1 para uso agrario, Tabla 2 para conservación y Tabla 3 para uso urbano.

Tabla 1. Metas establecidas para identificar suelo apto para uso agrario.

\begin{tabular}{|l|l|}
\hline \multicolumn{1}{|c|}{ Intención } & \multicolumn{1}{|c|}{ Descripción } \\
\hline Meta 1: AG1 & Identificar suelo físicamente apto para tierras de cultivo \\
\hline Meta 2: AG2 & Identificar suelo apto para el manejo intensivo de pastoreo \\
\hline Meta 3: AG3 & Identificar suelo apto para huertas de manzana \\
\hline
\end{tabular}

Tabla 2. Metas establecidas para identificar suelo apto para la protección permanente a través de la aplicación de estrategias de conservación.

\begin{tabular}{|c|l|}
\hline \multicolumn{1}{|c|}{ Intención } & \multicolumn{1}{|c|}{ Descripción } \\
\hline Meta 1: CO1 & Identificar suelo apto para la protección de biodiversidad nativa \\
\hline Meta 2: CO2 & $\begin{array}{l}\text { Identificar suelo apto para la protección de superficies y } \\
\text { calidad de agua }\end{array}$ \\
\hline Meta 3: CO3 & $\begin{array}{l}\text { Identificar suelo apto para la protección de importantes pro- } \\
\text { cesos ecológicos }\end{array}$ \\
\hline Meta 4: CO4 & $\begin{array}{l}\text { Identificar suelo apto para la recreación basados en los re- } \\
\text { cursos }\end{array}$ \\
\hline Meta 5: CO5 & $\begin{array}{l}\text { Identificar suelo apto para la mejora de áreas de con- } \\
\text { servación existente }\end{array}$ \\
\hline Meta 6: CO6 & $\begin{array}{l}\text { Identificar suelo con valores aptos para la protección per- } \\
\text { manente a través de la aplicación de estrategias de con- } \\
\text { servación }\end{array}$ \\
\hline
\end{tabular}

Tabla 3. Metas establecidas para identificar suelo apto para el desarrollo urbano.

\begin{tabular}{|l|l|}
\hline \multicolumn{1}{|c|}{ Intención } & \multicolumn{1}{c|}{ Descripción } \\
\hline Meta 1: UR1 & Identificar suelo apto para uso residencial \\
\hline Meta 2: UR2 & Identificar suelo apto para uso comercial \\
\hline Meta 3: UR3 & Identificar suelo apto para uso comercial al por menor \\
\hline Meta 4: UR4 & Identificar suelo apto para uso industrial \\
\hline
\end{tabular}

\subsubsection{Inventario de datos}

Los datos para el análisis de uso de suelo y la toma de decisiones fueron agrupados dentro de siete categorías descritas por Ian McHarg en Design with Nature (McHarg, 1969), en las que se consideran el subsuelo y el suelo como sustratos sobre las que ocurre la vegetación, vida animal, vida humana, y todas las asociaciones entre la sociedad y la naturaleza: 
a. Geofísico. Elementos abióticos de las características nativas, incluyendo geología, suelos, hidrología y clima.

b. Biológico-Ecológico. Elementos bióticos de las características nativas, incluyendo vegetación, hábitat animal, distribución de especies y diversidad.

c. Demográfico. Datos que describen las poblaciones humanas y su distribución.

d. Económico. Datos que describen la propiedad de la tierra, tendencia de los costos, propiedad de parcelas, valor de mercado por hectárea, valor por parcelas y años de construcción.

e. Político. Datos derivados de zonificación de distritos, planes, límites de la ciudad, límites de población, distritos de manejo de aguas, planeación regional y suelos de conservación.

f. Cultural. Datos que capturan la distribución y registro de sitios históricos y culturales.

g. Infraestructura. Datos que representan la distribución espacial y características de la infraestructura física necesaria para el soporte de los asentamientos humanos tales como carreteras, vías de ferrocarril, servicios, áreas comerciales.

\subsubsection{Idoneidad}

La modelación de la idoneidad para usos de suelo es un proceso analítico que determina la aptitud de una unidad del suelo o para un uso específico. La premisa fundamental del modelo depende de los diferentes valores y motivaciones de los grupos que representan los intereses. Por ello se establece la Unidad de Asignación Sencilla (SUA) y la Unidad de Asignación Múltiple (MUA), por sus siglas en inglés.

La SUA mide la idoneidad de cada unidad empleando un valor de rangos del 1 al 9, donde 1 representa baja idoneidad y 9 representa alta idoneidad. El proceso técnico consiste en determinar el nivel de medición de las características dentro de una capa ráster individual. Posteriormente se requiere seleccionar los métodos apropiados para la asignación de utilidad, convertir el formato ráster del dato original en formato vectorial $\mathrm{y}$, finalmente, asignar valores de utilidad para las características individuales dentro de la capa ráster usando reclasificaciones. La MUA combina múltiples capas de SUA a partir de una combinación ponderada, donde cada capa de entrada es asignada representando la idoneidad, equivaliendo la suma de todas las capas al 100\%, mientras que se aplica una sentencia condicional como un criterio para representar un orden de asignación de cada idoneidad, ya sea alta o baja.

Ambas unidades de asignación son generadas con procesos de evaluación espacial multicriterio, procesos que combinan y transforman los datos geográficos en una decisión de acuerdo con las preferencias especificadas. De esta forma fue posible evaluar diferentes alternativas para plantear una solución a un problema de decisión espacial, además de generar escenarios para disminuir la incertidumbre en relación con la toma de decisiones y evaluar las alternativas (Daga, 2009). 
El total de modelos generados en el Model Builder y Arc Tolbox de ArcMap a partir de los sub-objetivos, objetivos y metas planteadas fueron 102. Se agruparon en agrario, conservación y urbano, éste último dividido en cuatro grupos: residencial, comercial, industrial y comercial al por menor.

\subsubsection{Preferencia}

Para transformar la idoneidad en preferencia se utilizan diversos grupos de participantes que representan sectores de la comunidad con diferentes intereses. En este caso se optó por elegir cuatro grupos representativos de la comunidad en Cuauhtémoc: Gobierno, sociedad civil, sector económico y sector académico, ya que son los grupos que mayor influencia tienen en la toma de las decisiones en la ciudad. Se aplicó una encuesta de preferencia de usos de suelo entre estos cuatro grupos de usuarios. Las encuestas se realizaron a través de internet a un total de 124 encuestados y de forma aleatoria. A partir de las tres categorías de usos de suelo, se realizaron pares de comparaciones.

Los datos se organizaron mediante un proceso de jerarquía analítica (Analytical Hierarchy Process-AHP), el cual provee mediciones de coherencia, deriva prioridades entre criterios y alternativas, además de simplificar clasificaciones de preferencia entre los criterios de decisión a partir de los pares de comparaciones (Bunruamkaew, 2012).

\subsubsection{Conflicto}

Ya que el análisis se basó en tres categorías de uso de suelo, la caracterización del conflicto entre estas categorías se determinó mediante tres modelos tipo ráster que expresan las preferencias de uso de suelo, combinadas y reclasificadas dentro de áreas en conflicto y áreas de no conflicto. El conflicto ocurre cuando: 1) tres celdas comparten la misma localización espacial y tienen igual valor de preferencia (mayor conflicto); 2) cuando dos celdas tienen igual valor de preferencia y la tercera tiene un valor más bajo (conflicto moderado).

\subsection{Asignación futura de usos de suelo}

El modelo es capaz de identificar el conflicto potencial entre usos de suelo. Estas superficies de conflicto pueden ser utilizadas para muchas aplicaciones. Una de esas es la planificación futura de los usos de suelo en la asignación de escenarios, que facilitan la gestión de crecimiento inteligente (Carr y Zwick, 2007). Para ello se generó en la zona de estudio una asignación de línea base, a partir del estatus de las políticas y densidad urbana establecidas en el Plan de Desarrollo Urbano de Ciudad Cuauhtémoc. Para el desarrollo fue necesario tener en cuenta el incremento de población proyectada, la densidad urbana, el número total de hectáreas urbanas requeridas para soportar la población proyectada y, finalmente, el modelo de conflictos y preferencia de uso de suelo generada a partir de LUCIS. 


\section{Resultados}

\subsection{Definición y asignación de idoneidad del uso de suelo}

Para evaluar la idoneidad de uso en las categorías de agricultura, conservación y urbano, fue necesaria la aplicación de varios objetivos y subobjetivos en la conformación de cada una de las metas. En la categoría de agricultura se establecieron tres metas. La primera meta (AG1) consistió en la idoneidad para tierras de cultivo, donde se utilizaron datos de suelo física y económicamente aptos, distribuidos principalmente al norte y oeste de la zona urbana (Fig. 2). Para la segunda meta en agricultura (AG2), las áreas con mayor idoneidad para el manejo intensivo de pastoreo se identificaron al sur y al este de la zona de estudio (Fig. 2), sobre áreas naturales, ya que son las únicas que cuentan con coberturas de pastizales y bosque de encino, mientras que algunas zonas agrarias tienen aptitud moderada. La mayoría de los predios de la zona tienen aptitud territorial para el desarrollo de huertas de manzana, con una especial concentración en la zona norte de la ciudad (Fig. 2), estableciendo a partir de este uso la idoneidad agraria en la tercera meta (AG3).

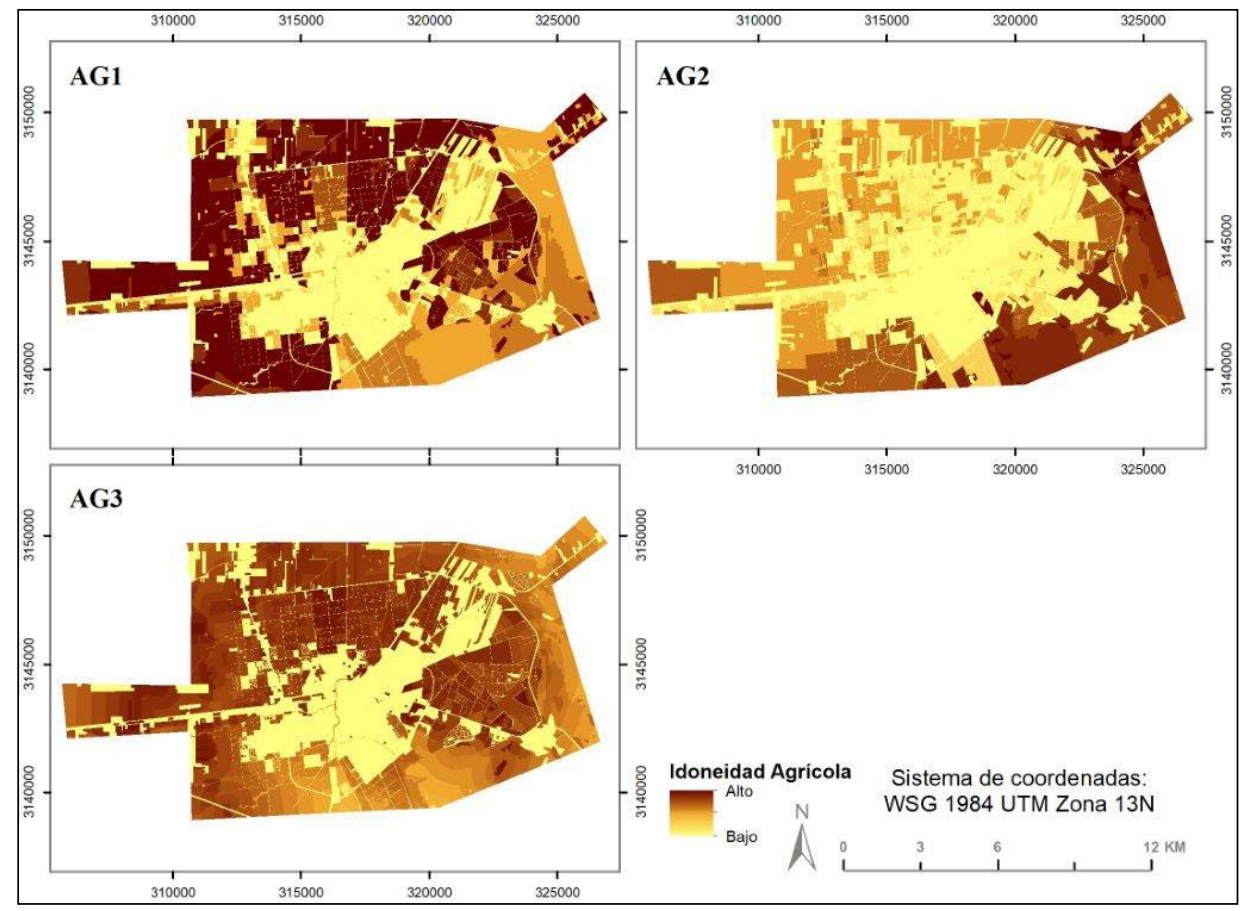

Figura 2. Idoneidad Agraria. AG1) Tierras de cultivo. AG2) Manejo intensivo de pastoreo. AG3) Huertas de manzana.

En la categoría de conservación se identificaron áreas idóneas a partir de seis metas. La primera meta (CON1) consistió en identificar suelo apto para la protección 
de biodiversidad nativa (Fig. 3). Se estableció a partir de la identificación de hábitats de humedales prioritarios, hábitats estratégicos para áreas de conservación, puntos importantes de biodiversidad, además de suelo que tuviera baja densidad de caminos y áreas de conservación existente. La zona de alta idoneidad para CON1 se localizó en la región sureste.

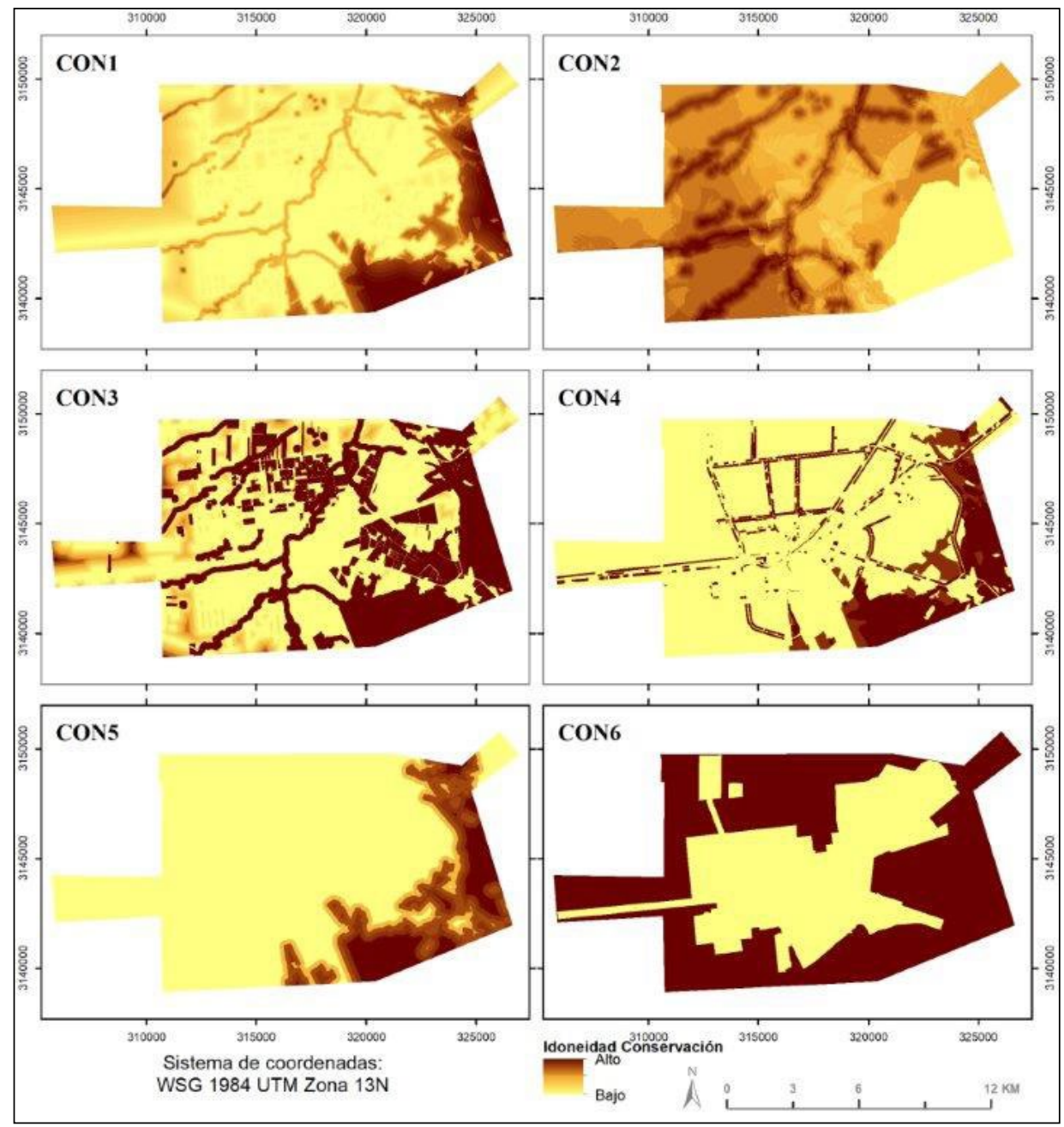

Figura 3. Idoneidad Conservación. CON1) Protección de biodiversidad nativa. CON2)

Protección de superficies y calidad de agua. CON3) Protección de importantes procesos ecológicos. CON4) Protección basada en los recursos existentes. CON5) Mejoramiento de áreas de conservación existente. CON6) Suelo con valor apto para la protección permanente.

La segunda meta en conservación (CON2) (Fig. 3) consistió en identificar suelo apto para la protección de superficies y calidad de agua. Incluye suelo con alta ca- 
pacidad de recarga potencial y acuíferos vulnerables, áreas de influencia asociadas a la infiltración de suelos adyacentes, así como ríos, lagos y estanques con un área de influencia que permita la infiltración. Las zonas con mayor idoneidad para la protección de biodiversidad nativa se encuentran al este y sureste, en su mayoría a lo largo de cauces y alrededor de cuerpos de agua. Región que se localiza en serranías con coberturas vegetales como bosques de encino, matorrales y pastizales, con una gran concentración de especies nativas.

La tercera meta en conservación (CON3) (Fig. 3) consistió en identificar suelo apto para la protección de importantes procesos ecológicos. Entre estos procesos se incluyen comunidades que pueden ser perjudicadas por el fuego, áreas no vulnerables para la protección contra el fuego, suelo importante para mantener el proceso de alimentación y almacenamiento de alimentos en el paisaje, además de ríos y cuerpos de agua con áreas de influencia suficientes para la protección de la función de la alimentación.

En la cuarta meta (CON4) (Fig. 3), se identificaron áreas aptas para la recreación basada en los recursos. Aquí se incluyeron parques y áreas recreativas, sitios históricos y culturales, superficies de agua con potencial para uso de recreación, infraestructura linear existente para el uso de corredores, y hábitats nativos aptos para el ocio. Los resultados en CON4 demuestran que la zona de bosque al sur de la ciudad es ideal para la conservación. También se localizaron importantes áreas potenciales a partir de corredores urbanos que se generan sobre las vialidades principales y la vía del ferrocarril.

En la quinta meta (CON5) (Fig. 3), se identificó suelo apto para la mejora de áreas de conservación existentes, particularmente áreas conectadas y suelo cercano a las áreas naturales existentes. De acuerdo con los resultados obtenidos, en la zona existen pocas áreas con alta idoneidad para ser mejoradas, ya que se encuentran fuertemente afectadas por las actividades humanas, tanto en la cercanía con la zona urbana como en la zona agraria. Mejorar las áreas de conservación es fundamental, ya que son recursos que deben mantenerse de forma radical. Sin embargo, el tipo de propiedad en el que se encuentran, ya sea ejidal o privada, no favorece la aplicación de estrategias para la mejora y conservación.

La última meta en conservación (CON6) (Fig. 3) consistió en identificar suelo con valores aptos para la protección permanente a través de la aplicación de estrategias de conservación, utilizando las áreas establecidas en el Plan de Desarrollo Urbano como zonas de reserva. La cartografía únicamente muestra dos rangos de clasificación: en el rango bajo se localizaron las zonas urbanizadas y las que se pretende urbanizar; por el contrario, la zona de alta aptitud para la protección permanente cubre tanto zonas de conservación en bosques como pastizales y áreas agrarias. Es importante mencionar que la protección de las zonas agrarias favorece el mantenimiento de la producción de alimentos de una ciudad, así como la estabilidad en su economía, como ocurre en el caso de Cuauhtémoc, donde las huertas de manzana son una de las principales fuentes de trabajo en la ciudad. 
Para la categoría de urbano se establecieron cuatro metas generales. La primera (UR1) consistió en identificar suelo tanto física como económicamente apto para uso residencial. En esta categoría se incluyeron áreas libres de inundación y ruido, alejadas de sitios con residuos peligrosos, próximas a desarrollos residenciales, escuelas, hospitales, carreteras, parques, sitios históricos y culturales, con servicios públicos y con valores catastrales adecuados para el uso residencial. El mapa de idoneidad para uso residencial UR1 (Fig. 4) demuestra que existen zonas aptas para nuevos desarrollos, situadas principalmente en la zona norte y suroeste de la ciudad, concentrándose en las cercanías de las vías principales y zonas residenciales existentes.

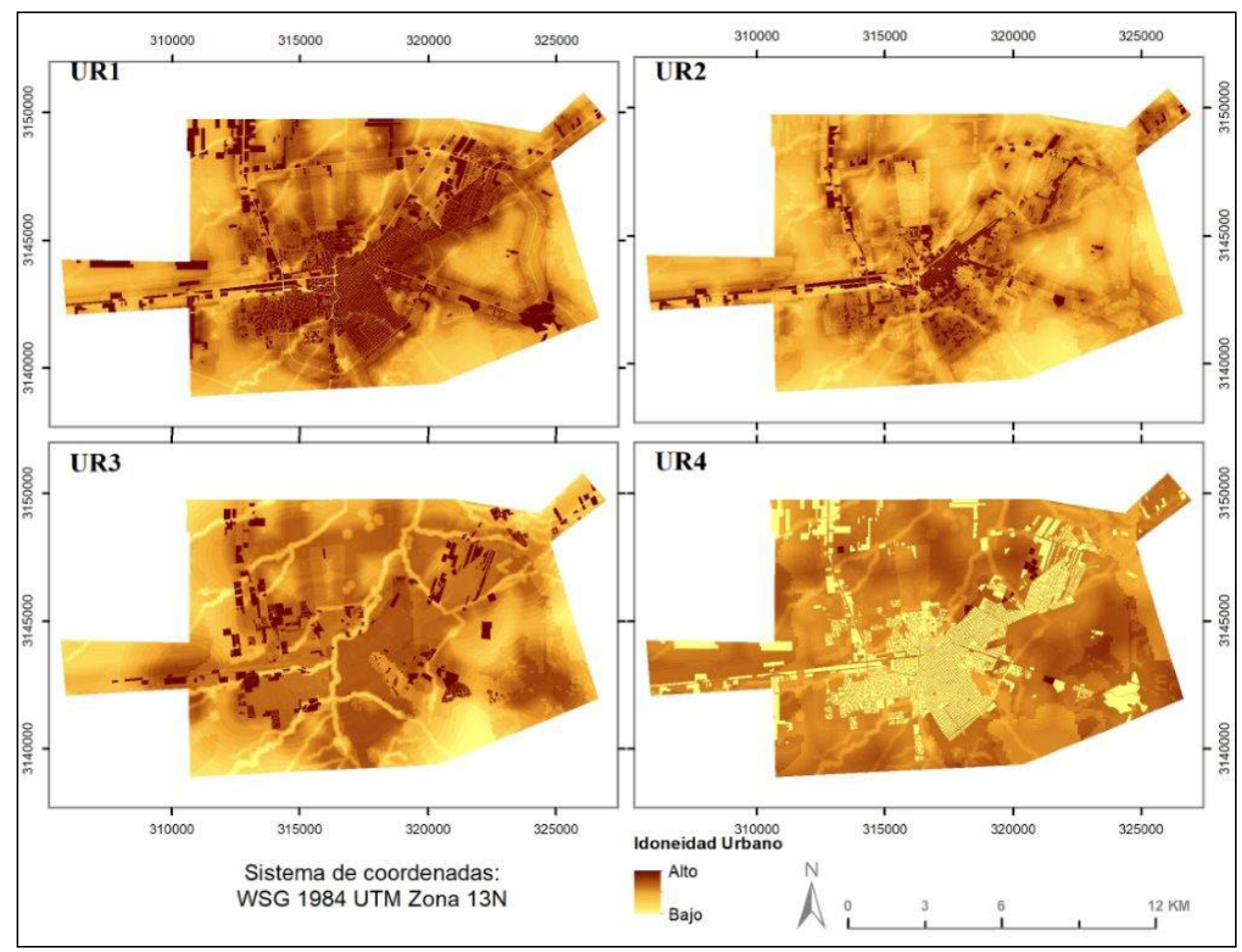

Figura 4. Idoneidad Urbana. UR1) Uso residencial. UR2) Uso comercial. UR3): Uso comercial al menudeo. UR4) Uso industrial.

En la segunda meta (UR2) (Fig. 4) se identificaron áreas aptas para uso comercial, con variables similares a las aplicadas en el uso residencial. Al combinar los elementos físicos y económicos, en el área de Ciudad Cuauhtémoc no existe gran cantidad de lugares con una idoneidad alta para el uso comercial y de oficinas. Los lugares que cuentan en el presente con alguna actividad, como es el caso de las áreas comerciales, se consideran ya altamente idóneos para el uso de suelo que ya están desarrollando. 
La tercera meta en urbano (UR3) (Fig. 4) consistió en identificar suelo física y económicamente apto para uso comercial Fig. por menor además de aplicar la condicional de asignar una idoneidad alta para todos los predios en condición de baldío, ya que son estos los que tienen mayor factibilidad para venderse o presentar un cambio de uso de suelo por su inactividad. De esa forma se identificaron todas aquellas áreas con potencial para el uso comercial al por menor en Ciudad Cuauhtémoc, donde existen predios baldíos distribuidos por toda el área de estudio, principalmente al oeste de la ciudad.

Finalmente, en la cuarta meta en urbano (UR4) (Fig. 4), en relación con el suelo para uso industrial, los resultados mostraron que en Cuauhtémoc existen pocas áreas con aptitud para uso industrial. La mayor idoneidad coincide con sitios alejados de las áreas residenciales y más accesibles a vías de comunicación. También el valor catastral del suelo fue uno de los elementos que más influyeron.

\subsection{Preferencia}

La encuesta de preferencia de usos de suelo entre los cuatro grupos de usuarios en Cuauhtémoc se aplicó a un total de 124 encuestados. De éstos, en su mayoría originarios del municipio de Cuauhtémoc, Chihuahua, el 25\% pertenecen al sector gobierno; el $8,87 \%$ son miembros de la sociedad civil; el $27,4 \%$ son del sector productivo, y el $41,1 \%$ del sector académico. La distribución interna de la muestra no es proporcional, debido a que la encuesta fue desarrollada en un sistema de captura a través de internet. Sin embargo, se consideran válidos los datos ya que son representativos para los objetivos del modelo.

Con relación al modelo para la estrategia de identificación de los conflictos de uso de suelo, se establecieron pares de comparaciones de acuerdo con las metas establecidas para la categoría agrario, urbano y conservación. Los usuarios debían establecer si uno era más importante que otro, o bien tenían una misma importancia en un rango de valores del -9 al 9 , en donde los valores encontrados entre -1 y 1 significan que son de igual importancia. Como resultado se identificaron aspectos relevantes, por ejemplo, las cuestiones de acceso al agua sobre cualquier otro elemento de los analizados.

Dentro de la categoría agrario, las tierras de pastoreo resultaron menos relevantes, mientras que las huertas de manzana y las tierras de cultivo se identificaron con igual importancia. En la categoría de conservación destaca la importancia del agua y la biodiversidad, mientras que los sitios de recreación no fueron considerados relevantes. Finalmente, en la categoría urbano, se destaca la importancia de la zona comercial y residencial, mientras que las áreas en desuso como predios baldíos, no son de relevancia para los habitantes de la localidad (Tabla 4). 
Tabla 4. Ponderación final de preferencia por los usuarios de Ciudad Cuauhtémoc.

\begin{tabular}{|c|c|}
\hline Idoneidad & $\begin{array}{c}\text { Ponderación de } \\
\text { preferencia }\end{array}$ \\
\hline \multicolumn{2}{|l|}{ AGRARIO } \\
\hline Agrario Meta 1: Tierras de cultivo & $45,4 \%$ \\
\hline Agrario Meta 2: Tierras de pastoreo & $15,7 \%$ \\
\hline Agrario Meta 3: Huertas de manzana & $38,9 \%$ \\
\hline Total & $100 \%$ \\
\hline \multicolumn{2}{|l|}{ CONSERVACIÓN } \\
\hline Conservación Meta 1: Biodiversidad & $11,0 \%$ \\
\hline Conservación Meta 2: Agua & $42,0 \%$ \\
\hline Conservación Meta 3: Procesos ecológicos & $15,9 \%$ \\
\hline Conservación Meta 4: Áreas recreativas & $4,9 \%$ \\
\hline Conservación Meta 5: Áreas de conservación & $16,4 \%$ \\
\hline Conservación Meta 6: Suelo de reserva & $9,7 \%$ \\
\hline $\begin{array}{rr}\text { Total } \\
\end{array}$ & $100 \%$ \\
\hline \multicolumn{2}{|l|}{ URBANO } \\
\hline Urbano Meta 1: Residencial & $28,1 \%$ \\
\hline Urbano Meta 2: Comercial & $42,7 \%$ \\
\hline Urbano Meta 3: Baldíos & $7,0 \%$ \\
\hline Urbano Meta 4: Industrial & $22,2 \%$ \\
\hline Total & $100 \%$ \\
\hline
\end{tabular}

\subsection{Conflicto}

El quinto paso para la integración del modelo consistió en la identificación del potencial de conflicto entre usos de suelo. Para esto fue necesario, primero, la selección de todas aquellas zonas que no podrían generar un conflicto en el futuro ya que no pueden cambiar de uso. Se trata de áreas urbanas ya consolidadas, como las zonas residenciales, comerciales e industriales, además de cuerpos de agua y arroyos. El modelo LUCIS considera excluir las áreas con suelos de conservación existentes. Sin embargo, para la región de estudio no existen áreas naturales protegidas y, por lo tanto, no pueden ser consideradas como zona de exclusión, ya que en el futuro sí puede presentarse un cambio de uso de suelo que generará conflictos con otros usos.

En la cartografía de preferencias y conflictos entre coberturas y uso de suelo (Fig. 5), se pudo observar que gran parte del conflicto se encuentra entre los límites del área urbana y la zona agraria. Esto corresponde a 2918 ha que pueden convertirse en uso urbano o bien seguir bajo uso agrario. El mayor conflicto se encuentra en la zona sur y sureste de la ciudad, en donde existen predios con alta aptitud urbana, agraria y de conservación al mismo tiempo; se trata de zonas de pastizales que pueden ser transformadas en predios agrarios o fraccionamientos, o bien seguir siendo pastizales. La cuestión radica en el tipo de propiedad que existe en esas áreas, ya que tienen un régimen privado en el que los intereses personales influyen de manera diferente a las zonas con propiedad ejidal. 


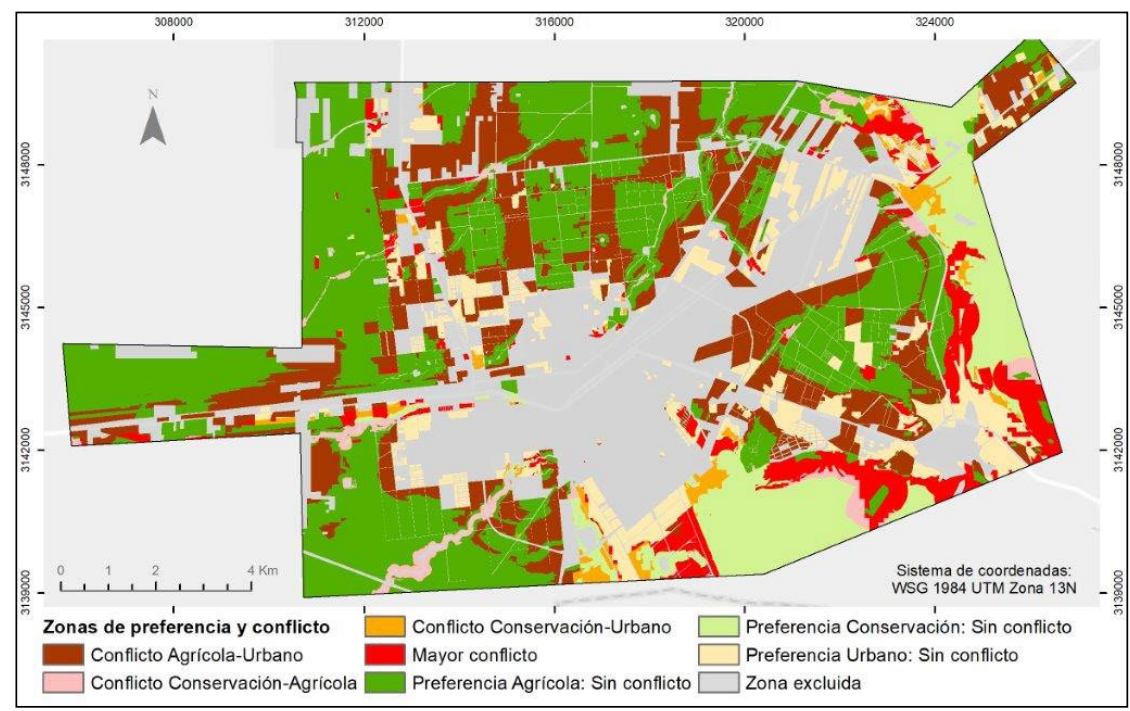

Figura 5. Áreas de preferencia y conflicto potencial de coberturas y usos de suelo en Ciudad Cuauhtémoc.

\subsection{Asignación futura de uso y cobertura de suelo para la zona de estudio}

El primer elemento requerido fue la proyección de la población futura (CONAPO, 2016). Para Ciudad Cuauhtémoc se estima en un total de 149.139 habitantes hacia el año 2030 (Fig. 6). Tomando en cuenta lo establecido en el Plan de Desarrollo Urbano de Cuauhtémoc y los datos poblacionales actuales (INEGI, 2015), existe una densidad de 40,47 hab/ha en el año 2015, y se pretende generar una densidad de hasta 50 hab/ha para el año 2030 y mantenerla en ese rango.

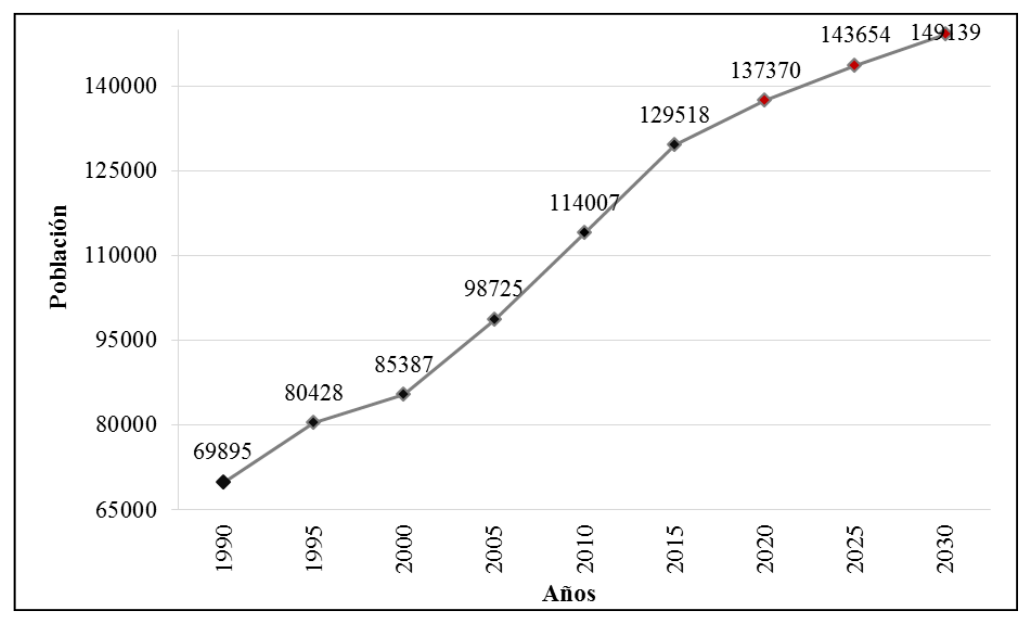

Figura 6. Crecimiento y población proyectada (1990-2030) para Ciudad Cuauhtémoc, Chihuahua. Fuente: (INEGI, 2015; CONAPO, 2016). 
El incremento poblacional representa 19.621 habitantes entre 2015 y 2030, por lo que fue necesario conocer la cantidad de hectáreas requeridas para albergar ese crecimiento. Para ello se utilizó la ecuación fundamental de uso de suelo regional (Carr y Zwick, 2007), que utiliza la población y la densidad urbana requerida: Esto representa 392,42 hectáreas adicionales requeridas para el crecimiento urbano.

El procedimiento realizado para establecer la asignación de futuros usos de suelo fue posible de acuerdo con el desarrollo de idoneidad a través del rol de los expertos, análisis territorial y preferencia de los usuarios en la comunidad para lograr realizar la identificación potencial de los conflictos y visualizar el potencial del uso de suelo a futuro (Carr y Zwick, 2007).

En un balance general, el área de estudio cuenta con 16.272,21 ha, de las cuales $38,38 \%$ se asignaron a la categoría de agricultura, 11,95\% a conservación y 49,67\% a urbano (Tabla 5). Existe una porción importante del área que se encuentra en conflicto entre los usos de alguna de las tres metas, por lo que las conveniencias productivas de la región y la necesidad de nuevos espacios son los que pueden establecer la forma en que los usos de suelo puedan cambiar (Fig. 7).

Tabla 5. Tabulación de la asignación de usos de suelo a futuro para el año 2030. Caso de estudio de Ciudad Cuauhtémoc de acuerdo con los principales análisis del modelo LUCIS.

\begin{tabular}{lrr}
\hline \multicolumn{1}{c}{ Asignación de uso de suelo } & Hectáreas & $\begin{array}{c}\text { \% de } \\
\text { área }\end{array}$ \\
\hline AGRARIO & & \\
$\begin{array}{l}\text { Donde la preferencia agraria reclasificada excede la preferencia de con- } \\
\text { servación y urbano (Áreas sin conflicto) }\end{array}$ & 4815,12 & $29,59 \%$ \\
$\begin{array}{l}\text { Donde la preferencia agrario original excede las preferencias de con- } \\
\text { servación y urbano, pero la preferencia reclasificada indica conflicto }\end{array}$ & 1429,52 & $8,79 \%$ \\
Subtotal agrario & $\mathbf{6 2 4 4 , 6 4}$ & $\mathbf{3 8 , 3 8 \%}$ \\
\hline
\end{tabular}

\section{CONSERVACIÓN}

Donde la preferencia en conservación reclasificada excede la preferencia agrario y urbano (Áreas sin conflicto)

$1306,16 \quad 8,03 \%$

Donde la preferencia de conservación original excede las preferencias de agricultura y urbano, pero la preferencia reclasificada indica conflicto

Suelos de conservación existentes

$469,32 \quad 2,88 \%$

Cuerpos de agua y arroyos existentes

$0 \%$

Subtotal conservación

$168,97 \quad 1,04 \%$

\section{URBANO}

Donde la preferencia reclasificada en urbano excede la preferencia agraria y

de conservación (Áreas sin conflicto)

$1944,45 \quad 11,95 \%$

Donde la preferencia urbana original excede las preferencias de agricultura

y de conservación, pero la preferencia reclasificada indica conflicto

Áreas urbanas existentes

$1206,45 \quad 7,41 \%$

Calles, carreteras y caminos

$2339,92 \quad 14,38 \%$

$3106,41 \quad 19,09 \%$

Subtotal urbano

$1430,34 \quad 8,79 \%$

TOTAL

$\mathbf{8 0 8 3 , 1 2} 49,67 \%$

$16.272,21 \quad 100 \%$




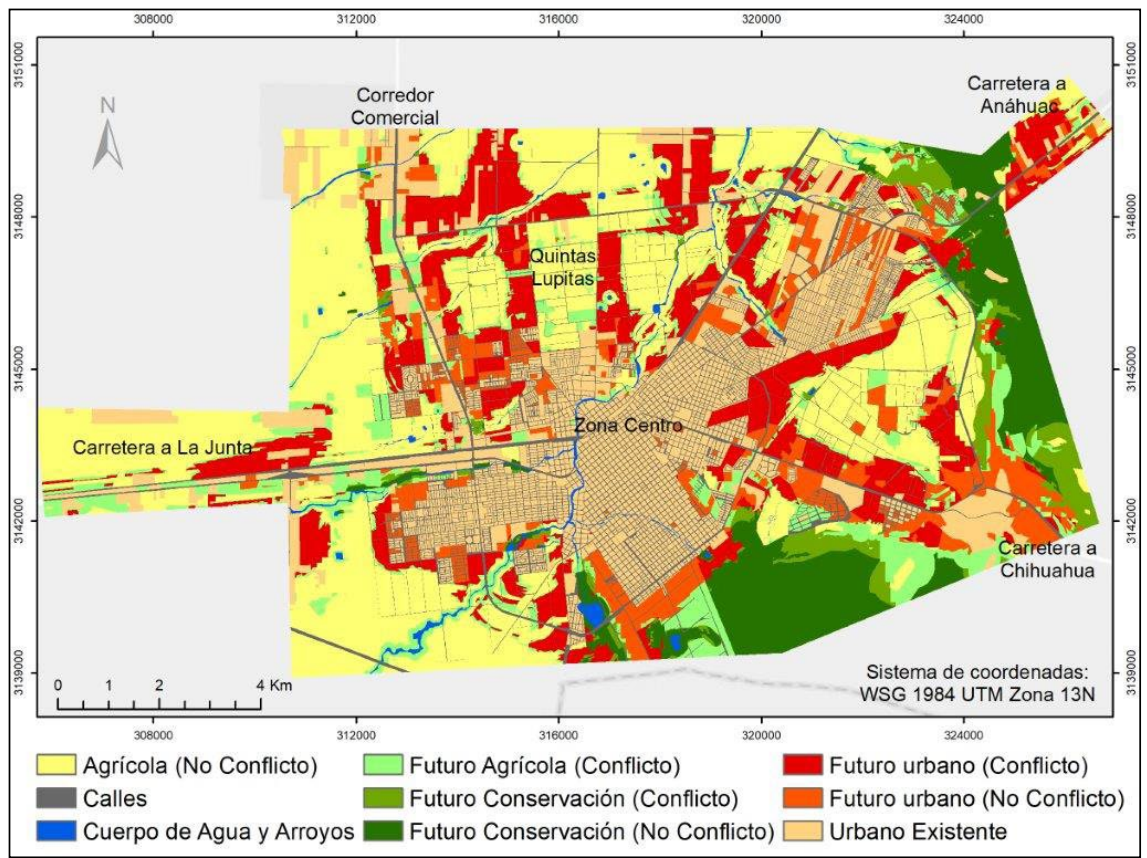

Figura 7. Asignación de uso de suelo para el año 2030 en Ciudad Cuauhtémoc, Chihuahua.

La asignación de usos de suelo para el año 2030 en la ciudad presenta una diversidad de conflictos en el uso y cobertura de suelo. Sin embargo, para un análisis más en detalle es necesario identificar el tipo de propiedad y las preferencias que tiene cada propietario para desarrollar cierta actividad, ya que son quienes tienen la decisión final, con excepción de aquellas zonas en donde exista alguna ley que pueda establecer lo contrario (Fig. 7). Por ejemplo, la carretera a Anáhuac demuestra el potencial urbano futuro, aunque se presentan gran cantidad de predios en conflicto con la zona agraria; la zona del Corredor Comercial se considera una zona de conflicto potencial entre las actividades agrarias y el posible desarrollo urbano; mientras que la región de Las Quintas Lupitas sigue prevaleciendo como zona agraria; en la zona poniente y centro-sur de la zona siguen prevaleciendo los usos de suelo de pastizales y bosque de encino; la Carretera a La Junta presenta conflictos para el desarrollo urbano, ya que la mayoría es de asignación agraria. Existen varias zonas en los límites de la ciudad que no presentan conflictos para la expansión urbana.

\section{Discusión}

El análisis de conflictos de usos de suelo y la delimitación del límite de expansión urbana ofrece las bases de información necesarias para que los planificadores elaboren las propuestas de política pública para un crecimiento urbano que permita el equilibrio entre el potencial del territorio y las preferencias de uso de acuerdo con las vocaciones productivas de la región. A pesar de las problemáticas actuales por el cambio de cober- 
tura y uso de suelo, como la pérdida del área agraria y natural en Ciudad Cuauhtémoc, pareciera que no existe regulación en el proceso de crecimiento urbano.

Los planificadores de la ciudad deben asumir un compromiso para el desarrollo de ésta, de forma particular en actividades que permitan formar sinergias entre la sociedad, el medio ambiente y los sistemas económicos, ya que el crecimiento desordenado afecta a todos ellos (Loya et al., 2015). De acuerdo con los resultados de esta investigación se estableció la idoneidad para las actividades agrarias, urbanas y de conservación, tomando en cuenta las preferencias de los usuarios e identificando los conflictos de usos de suelo para las asignaciones de futuros usos. El modelo muestra que la suburbanización en ciudades como Cuauhtémoc es inevitable debido al rápido incremento poblacional y al desarrollo económico.

De acuerdo con la problemática establecida para el caso específico de Ciudad Cuauhtémoc, Chihuahua, es necesario establecer medidas de prevención contra el crecimiento disperso de la ciudad y evitar los conflictos de uso de suelo, ya que estudios previos indican que puede generar altos costos. Actualmente las ciudades medias tienen la posibilidad de explorar diversas alternativas para evitar los problemas generados en otras ciudades donde la expansión urbana ya está presente. Por ello aplicar estrategias de identificación de conflictos en los usos de suelo es una medida eficaz para poder dar certidumbre al crecimiento y desarrollo de diversas poblaciones.

Basta decir que, en el año 2017, las autoridades locales han promulgado la conformación de un Consejo de Planeación Urbana Municipal (COPLUM), donde por primera vez en la historia de Ciudad Cuauhtémoc se tiene contemplado el inicio de funciones del Instituto Municipal de Planeación (IMPLAN).

\section{Conclusiones}

La utilización del modelo LUCIS ha resuelto de forma satisfactoria el objetivo planteado para esta investigación, ya que ha sido posible la identificación de áreas con la mayor aptitud territorial para albergar un crecimiento urbano equilibrado, a partir de un análisis de conflictos entre la capacidad física del suelo, áreas urbanas, agrarias y de conservación. Los resultados han permitido entender el significado espacial del estatus de las políticas de uso de suelo, incluyendo los patrones probables del uso de suelo en el futuro asociados con las tendencias agrarias, urbanas y de conservación. El modelo permite además incluir el acomodo del incremento de la población proyectada para el futuro y la protección de suelos productivos, tanto agrarios como medioambientales.

El Plan de Desarrollo Urbano vigente plantea ciertas áreas para la expansión de Ciudad Cuauhtémoc, las cuales son validadas por el modelo. Sin embargo, existen zonas en las cuales no es recomendable la construcción de nuevos fraccionamientos, por lo que el modelo desarrollado en la presente investigación propone nuevas áreas, que por lo general se establecen en los límites de la ciudad existente y cercanas a vías principales. Se consideraron áreas no solo para el futuro desarrollo urbano en la ciudad, sino que también se encontraron áreas para crecimiento en poblaciones rurales y campos menonitas.

En el caso de estudio se estima un total de 149.139 habitantes para el año 2030, lo que representa la necesidad de 392,42 hectáreas adicionales para albergar el crecimiento 
poblacional. Para ello de las 16.272,21 hectáreas que tiene el límite de población, el 38\% se asignaron a la categoría de agraria, el $11,95 \%$ a suelos de conservación y el $49,67 \%$ a suelo urbano (incluyendo el área urbana existente). Existe una porción importante del área que se encuentra en conflicto entre los diferentes usos de suelo. Se puede concluir que la integración de un modelo de resolución de conflictos de cobertura y uso de suelo representa una solución práctica que contribuye a la mejora de los procesos de planificación del desarrollo urbano.

Los principales desafíos en materia de planificación y desarrollo urbano en ciudades como la abordada en el presente estudio se deben a un marco jurídico obsoleto, a la falta de una visión integral de desarrollo, al inadecuado ejercicio de instituciones rígidas, con debilidades de los gobiernos locales que tienen un marco temporal de actuación muy corto que no les permite establecer un seguimiento de los planes y programas establecidos. En el caso específico de Ciudad Cuauhtémoc, existe ya una gran cantidad de estudios que han logrado exponer distintas problemáticas y proponer alternativas relacionadas con su desarrollo territorial. Sin embargo, no ha existido el eco necesario para integrar nuevos elementos en la mejora y el desarrollo urbano de la ciudad.

\section{Referencias}

Alatorre, L.C., Díaz, R.E., Miramontes, S., Sánchez, E., Bravo, L.C. 2014. Spatial and temporal evolution of Cuauhtémoc aquifer static level during the years 1973, 1991 and 2000: A geographical approach. Journal Geographic Information Systems 6, 572-584. http://doi. org/10.4236/jgis.2014.65047.

Bazant, J. 2010. Expansión incontrolada y paradigmas de la planeación urbana. Espacio Abierto, 19 (3), 475-503.

Bunruamkaew, K. 2012. How to do AHP analysis in Excel. University of Tsukuba, Tsukuba, Japan.

Carr, M., Zwick, P. 2007. Smart Land-Use Analysis: the LUCIS model, Land-Use Identification Strategy. ESRI Press, 292 pp.

Cifuentes, P.A. 2009. Modelización de los factores de crecimiento urbano como aporte a la sostenibilidad.Estudio de caso: Manizales-Colombia. Revista Internacional de Sostenibilidad, Tecnología y Humanismo 4, 81-96.

CONAPO 2016. Consejo Nacional de Población.

da Cunha, J., Rodríguez, J. 2009. Crecimiento urbano y movilidad en América Latina. Revista Latinoamericana de Población 3 (4-5), 27-64.

Daga, R.A. 2009. Determinación de áreas con aptitud para la expansión urbana con fines de ordenamiento territorial aplicando el análisis espacial multicriterio: Caso cuenca baja del río Lurín. Tesis Doctoral. Universidad Nacional Mayor de San Marcos, Lima, Perú.

Davidoff, P., Reiner, T. 1962. A Choice Theory of Planning. Journal of the American Institute of Planners 28 (2), 103-115. http://doi.org/10.1080/01944366208979427.

Díaz, R.E., Bravo, L.C., Alatorre, L.C., Sánchez, E. 2014. Análisis geoespacial de la interacción entre el uso de suelo y de agua en el área peri-urbana de Cuauhtémoc, Chihuahua. Un estudio socioambiental en el norte de México. Investigaciones Geográficas 83, 116-130. http://doi. org/10.14350/rig.32694.

Garza, G. 2010. La transformación urbana de México, 1970-2020. En: Garza, G., Schteingart, M. (Coords.), Desarrollo Urbano y Regional, El Colegio de México, México D.F., pp. 31-86.

INEGI 2015. Censo de Población y Vivienda. 
Loya, J.O., Torres, M.E., Bravo, L.C., Alatorre, L.C. 2015. Análisis del cambio de cobertura y uso de suelo durante el periodo 1995-2011, EMC y autómatas celulares para la predicción del crecimiento urbano, el caso de Ciudad Cuauhtémoc, Chihuahua. En: L. Alatorre, M. Torres, H. Rojas, L. Bravo, L.Wiebe, F. Sandoval, E. López (Eds.), Geoinformática aplicada a procesos geoambientales en el contexto local y regional: teledetección y sistemas de información geográfica. UACJ, Ciudad Juárez, Chihuahua, México, pp.163-194.

McHarg, I. 1969. Design with nature. Doubleday/Natural History Press, New York.

Molero, E., Grindlay, A., Asensio, J.J. 2007. Escenarios de aptitud territorial y modelización cartográfica del crecimiento urbano mediante técnicas de evaluación multicriterio. Geofocus $7,120-147$.

Peña, S. 2016. Teoría de la planeación: el paradigma clásico y los paradigmas emergentes. Planeación urbana y regional. Ciudad Juárez, México, COLEF-UACJ, pp. 35-65.

Rionda, J. 2008. Distribución de la población y crecimiento urbano en México. Análisis Económico, 52 (23), 201-218.

SDUE Gob. Municipal de Cuauhtémoc 2009. Plan de Desarrollo Urbano de Centro de Población. Secretaría de Desarrollo Urbano y Ecología, Cd. Cuauhtémoc, Chihuahua, México

Silberstein, J., Maser, C. 2013. Land-Use Planning for Sustainable Development. CRC Press, $296 \mathrm{pp}$.

Wacquant, L. 2001. Parias urbanos. Marginalidad en la ciudad a comienzos del milenio. Ed. Manantial, Buenos Aires, 204 pp. 